\title{
Satellite Broadband Capacity-on-Demand: Dynamic Beam Illumination with Selective Precoding
}

\author{
Lin Chen, Eva Lagunas, Symeon Chatzinotas, Björn Ottersten \\ Interdisciplinary Centre for Security, Reliability and Trust (SnT), University of Luxembourg, Luxembourg \\ Email: \{lin.chen, eva.lagunas, symeon.chatzinotas, bjorn.ottersten\}@uni.lu
}

\begin{abstract}
Efficient satellite resource utilization is one of the key challenges in next generation high-throughput satellite communication system. In this context, dynamic coverage scheduling based on traffic demand has emerged as a promising solution, focusing system capacity into geographical areas where it is needed. Conventional Beam Hopping (BH) satellite system exploit the time-domain flexibility, which provides all available spectrum to a selected set of beams as long as they are not adjacent to each other. However, large geographical areas involving more than one adjacent beam may require full access to the available spectrum during particular instances of time. In this paper, we address this problem by proposing a dynamic beam illumination scheme combined with selective precoding, where only sub-sets of beams that are subject to strong inter-beam interference are precoded. With selective precoding, complexity at the groundsegment is reduced and only considered when needed. Supporting results based on numerical simulations show that the proposed scheme outperforms the relevant benchmarks in terms of demand matching performance.
\end{abstract}

Index Terms-Flexible satellite communication, dynamic beam illumination, selective precoding

\section{INTRODUCTION}

Satellite communication systems represent a fundamental element to deliver Information and Communication Technologies (ICT) services to all regions of the world at an affordable cost [1].

High-Throughput Satellite (HTS) systems are characterized by multi-beam coverage and the re-use of the available satellite spectrum. Typically, the spectrum is divided into "colors" corresponding to a specific frequency and polarization, in an attempt to overcome the inter-beam interference. Following the growing traffic demands, the next generation of satellite systems focus on increasing the capacity by reducing the size of the spot-beams such that the beam gain is increased [2]. However, dividing the coverage to smaller beams increases the probability of having under-utilized beams, i.e. beams where demand is low compared to the supplied capacity. In fact, the traffic requested by each beam may differ significantly due to geographic variations (and time variations as well when considering mobile user terminals) [3].

One of the trending areas of research and development recent years has been the satellite payload flexibility and how to deliver cost-competitive connectivity in response to evolving consumer demand and cost expectations [4]. Conventional Beam Hopping (BH) satellite systems exploit the time-domain flexibility to provide all available spectrum to a selected set of beams as long as they are not adjacent to each other [5].
In other words, the traditional "color" separation is replaced by time division multiplexing. The set of illuminated beams changes in each time-slot based on a time-space transmission pattern that is periodically repeated. As the number of spotbeams grows, BH appears to be a valid approach to provide service to extensive coverage areas. The illumination pattern design for conventional $\mathrm{BH}$ systems has been studied in [6][10]. While [6], [7] focused on heuristic iterative sub-optimal algorithms, [8], [9] considered genetic and simulated annealing algorithms respectively targeting global optimal solutions at the expenses of increased computational complexity. Finally, [10] proposed to integrate deep learning into the optimization procedure in order to accelerate the optimization procedure.

The inclusion of time-flexibility provided by $\mathrm{BH}$ is limited by the spatially isolation between beams required to ensure that the co-channel interference is kept to minimal. In some particular cases where the high-demand areas expand over multiple adjacent beams, it makes sense to illuminate a cluster of beams at the same time instance. This was the motivation of the preliminary study carried out in [11], where precoding was first combined with $\mathrm{BH}$ in order to mitigate the inter-beam interference caused between adjacent beams that are simultaneously activated. Subsequently, the work in [12] proposed the so-called Cluster-Hopping $(\mathrm{CH})$ concept, where the overall multibeam coverage area is split into a set of non-overlapping beam-clusters that are illuminated dwelling just long enough to fill the demand in each cluster. Within a cluster, precoding is used to mitigate the interference while inactive clusters are placed between active clusters as a physical barrier to avoid strong inter-cluster interference.

The strict cluster definition of [12] was shown to be problematic in adapting to different types of demand distributions and suggests that a more flexible dynamic beam illumination design allowing clusters of different shapes and sizes would facilitate the per-beam demand matching objective. In addition, the pragmatic approach and the naive design (designed assuming an interference-free scenario) combined with the limited evidence of results in [11] motivated this work.

In this paper, we propose a beam scheduling combined with selective precoding, where only sub-sets of beams that are subject to strong inter-beam interference are precoded. In particular, we formulate a Binary Quadratic Programming (BQP) problem where the main goal is to penalize the activation of adjacent beams while constraining the system to provide a specific supplied capacity per each beam in a given time- 


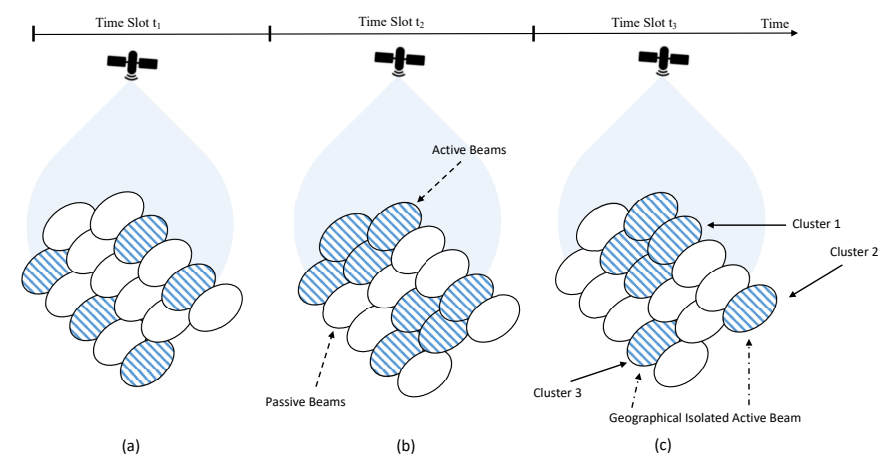

Fig. 1: Proposed Dynamic Beam Illumination Scheme

window. Whenever the solution includes strong inter-beam interference, precoding is applied to achieve an acceptable performance. Since precoding requires extensive computation, with selective processing, complexity at the ground-segment is reduced and only considered when needed.

The remainder of this paper is organized as follows. In Section II we present the system model. Section III focuses on the proposed dynamic beam illumination design. In Section IV we describe the proposed relaxation and solution. In Section V we provide supporting results based on numerical simulations. Finally, Section VI concludes the paper.

\section{SySTEM MODEL}

We consider the forward link of a multibeam geostationary (GEO) satellite communication system, where the payload is equipped with multiple-antenna elements which are able to generate a limited number of beams at each time slot. We assume that the satellite coverage area is divided into a specific number of possible virtual and fixed $N$ beams, among which the payload can simultaneously activate at most $K$ beams $(K<N)$. Single gateway with ideal feeder link and perfect user synchronization are assumed.

The beam average traffic demand is assumed to be known, and it is denoted as $D_{1}, \ldots, D_{N}$ (in bps). In order to deal with the expected high beam demands, all active beams reuse the same frequency band $B$. We assume that the satellite is required to satisfy the beams' demand within a time limit $T_{H}$. The time window $T_{H}$ is divided into $M$ time-slots of duration $T_{s}$ which denotes the minimum dwelling time of the hopping system. With the goal of matching demands with supplied capacity, we adopt dynamic beam scheduling and selective precoding. In particular, a maximum of $K$ beams can be activated simultaneously and, in high-demand geographical areas, those active beams may need to be adjacent to each other resulting in strong inter-beam interference. An example of the aforementioned situation is depicted in Fig. 1. To mitigate the inter-beam interference in case of adjacent beams, precoding is required to achieve an acceptable spectral efficiency. Therefore, precoding is only employed when needed.
The signal vector received by the $K$ users is denoted as $\mathbf{y} \in \mathbb{C}^{K \times 1}$ and further expressed as,

$$
\mathbf{y}=\mathbf{H x}+\mathbf{n}
$$

where $\mathbf{x} \in \mathbb{C}^{K \times 1}$ denotes the transmitted symbols; $\mathbf{H} \in$ $\mathbb{C}^{K \times K}$ refers to channel matrix, which is assumed to be perfectly known at the transmitter, and $\mathbf{n} \in \mathbb{C}^{K \times 1}$ denotes the zero-mean additive Gaussian noise. In particular, we assume $\mathbb{E}\left[\mathbf{n n}^{H}\right]=\sigma_{T}^{2} \mathbf{I}_{K}$, where $\sigma_{T}=\sqrt{\kappa T_{R x} B} ; \kappa$ denotes the Boltzmann constant and $T_{R x}$ is the clear sky noise temperature of the receiver.

The downlink channel matrix $\mathbf{H}$ between the satellite and $K$ users on Earth is modeled as,

$$
[\mathbf{H}]_{k, l}=\frac{\sqrt{G_{R}^{k} G_{k, l} e^{j \phi_{k, l}}}}{4 \pi \frac{d_{k}}{\lambda}}
$$

where $G_{R}^{k}$ is the $k$-th user's receiver gain; $G_{k, l}$ is the gain from $l$-th beam to the $k$-th user; $\phi_{k, l}$ represents a phase noise term resulting from the beam-pattern generation; $d_{k}$ is the distance between the satellite and $k$-th user; $\lambda$ denotes the wavelength.

The transmitted symbols $\mathrm{x}$ can be written as,

$$
\mathbf{x}=\mathbf{W} \mathbf{s}=\left[\begin{array}{lll}
\mathbf{W}_{1} & & \\
& \ddots & \\
& & \mathbf{W}_{C}
\end{array}\right] \mathbf{s}
$$

where s are the non-precoded symbols, with $\mathbb{E}\left[\mathbf{s s}^{H}\right]=\mathbf{I}_{K}$; and the selective-precoding matrix $\mathbf{W}$ of dimension $(K \times K)$ is block diagonal, with $\mathbf{W}_{i}$ refers to selective-precoding matrix for cluster $i,(i=1, \ldots, C)$. Especially the corresponding matrix $\mathbf{W}_{i}$ converts to: (i) The simple per-beam transmit power $P_{\text {beam }}$ for the clusters composed of a single active and geographically isolated beam; (ii) $\mathbf{0}$ value for each passive beams; (iii) For each cluster composed of more than one active beam, the precoding matrix follows the MMSE design [13], [14], which can be expressed as,

$$
\mathbf{W}_{i}=\eta \mathbf{H}_{i}^{H}\left(\mathbf{H}_{i} \mathbf{H}_{i}^{H}+\alpha \mathbf{I}\right)^{-1}
$$

where $\mathbf{H}_{i}$ is the channel matrix of all the users in cluster $i$, $\alpha$ is a predefined regularization factor, and $\eta$ is the power allocation factor which is expressed as,

$$
\eta=\sqrt{\frac{\left|\mathcal{K}_{i}\right| P_{\text {beam }}}{\text { Trace }\left(\mathbf{W}_{i} \mathbf{W}_{i}^{H}\right)}}
$$

where $\mathcal{K}_{i}$ denotes the number of users in cluster $i$.

\section{Dynamic Beam Illumination Problem}

Let us denote $x_{n, t} \in[0,1]$ as the binary assignment variable indicating that the $n$ beam is activated in time-slot $t$. As mentioned, the payload limits the number of simultaneously active beams, that is $\sum_{n=1}^{N} x_{n, t} \leq K, \forall t$. For convenience, let us denote $\mathbf{x}_{t}$ of dimension $(N \times 1)$ the indicator vector at time-slot $t$.

In this paper, the main objective is to avoid the complexity of precoding while satisfying the traffic demands. To address 
this problem, we propose a formulation that avoids the illumination of beams that generate strong interference to each other. In particular, we make use of a penalty matrix $\Omega$ of dimension $N \times N$ to punish this type of event. The considered optimization problem is detailed below as,

$$
\begin{aligned}
P_{0}: & \underset{\mathbf{x}_{1}, \ldots, \mathbf{x}_{M}}{\operatorname{minimize}} \sum_{t=1}^{M} \mathbf{x}_{t}^{T} \boldsymbol{\Omega} \mathbf{x}_{t} \\
\text { s.t. } & C_{1}: \sum_{n=1}^{N} x_{n, t} \leq K, t=1, \ldots, M \\
& C_{2}: R_{n} \geq D_{n}, n=1, \ldots, N \\
& C_{3}: x_{n, t} \in[0,1], t=1, \ldots, M ; n=1 \ldots, N
\end{aligned}
$$

where $R_{n}$ denotes the supplied capacity in [bps] to the $n$ th beam. There are three key aspects related with (6). First, the combinatorial nature of the problem due to the binary assignment variables $x_{n, t}$ render a non-convex difficult-tosolve problem. Second, the demand satisfaction constraint is difficult to operate as the supplied capacity $R_{n}$ depends on the beam activation configuration and the design of the precoders (in case that adjacent beams are activated). Finally, the definition of the interference penalty $\Omega$ plays a key role in the final solution of (6).

\section{A. Interference Penalty Matrix}

In this section, the model of $\Omega$ is discussed. Since interference is strongly correlated with the geographical distance, we propose a penalty matrix $\Omega$ based on the geographical separation of satellite beams. In order to model the geographical distribution of satellite beams on Earth, we make use of graph theory by considering the beam center as a vertex $v \in \mathcal{V}$ of a graph. Two vertices are connected with an edge if those vertices represent geographically adjacent beams. We assume an undirected unweighted graph, meaning that edge $e \in \mathcal{E}$ between vertices have the same weight which is fixed and equal to 1 . As a consequence, the graph is defined as $\mathcal{G}=(\mathcal{V}, \mathcal{E})$, and its adjacency matrix $\mathbf{A}$ is defined as the $N \times N$ matrix whose elements $A_{i, j}=1$ if $(i, j) \in \mathcal{E}$ and 0 otherwise. Note that the adjacency matrix $\mathbf{A}$ already contains the information related to geographical beam separation and, therefore, can be used as penalty matrix.

\section{B. Demand Satisfaction Constraint}

The actual supplied capacity $R_{n}$ is a function of the beam selection variable $\mathbf{x}_{t}, t=1, \ldots, M$ and, therefore, it is difficult to predict beforehand. Some works like [12] considered a limited set of illuminated beams configuration and assumed that the resulting $R_{n}$ was pre-computed and available at the resource management unit in charge of designing the illumination pattern. In the problem at hand, we do not wish to limit the search space and, as a consequence, the approach in [12] becomes computationally expensive, particularly for large number of beams. Other works like [11] considered an interference-free scenario to approximate the values of $R_{n}$.
To overcome this issue, we propose to convert the demand $D_{n}$ [bps] into approximated number of time-slots that a beam needs to be activated to satisfy such demand. We denote the latter as $\Delta_{n}, n=1, \ldots, N$, which is defined as,

$$
\Delta_{n}\left[\text { Number of } T_{s}\right]=\left\lceil\frac{D_{n}}{\zeta_{n}}\right\rceil
$$

where $\lceil x\rceil$ maps $x$ to the least integer greater or equal to $x$, and $\zeta_{n}$ denotes the average supplied capacity recorded in previous time-instances and measured in [bps]. The value of $\zeta_{n}$ can be computed at the satellite gateway based on the actual recorded delivered capacity of previous time-slots where beam $n$ was activated.

Using (7), the constraint $C_{2}$ in (6) can be reformulated as,

$$
C_{4}: \quad \sum_{t=1}^{M} \mathbf{e}_{n}^{T} \mathbf{x}_{t}=\Delta_{n}, n=1, \ldots, N
$$

where $\mathbf{e}_{n}$ denotes a vector with the $n$-th component equal to 1 and all others are 0 . As for the usage of ceil function, the inequality can be converted into equality.

\section{Problem Relaxation and Proposed Solution}

For the sake of simplicity, we compact our notation by rearranging all time-slots $t$ into a single tall vector $\mathbf{x}^{T}=$ $\left[\begin{array}{llll}\mathbf{x}_{1}^{T} & \mathbf{x}_{2}^{T} & \cdots & \mathbf{x}_{M}^{T}\end{array}\right]$. By defining $\tilde{\mathbf{A}}=\mathbf{I}_{M} \otimes \mathbf{A}$, being $\otimes$ denotes the Kronecker product, we can reformulate the objective function of (6) as $\mathbf{x}^{T} \tilde{\mathbf{A}} \mathbf{x}$. In particular, the resulting problem can be rewritten as,

$$
\begin{aligned}
P_{2}: & \underset{\mathbf{x}}{\operatorname{minimize}} \quad \mathbf{x}^{T} \tilde{\mathbf{A}} \mathbf{x} \\
\text { s.t. } & C_{5}: \mathbf{x}^{T} \mathbf{E}_{\mathbf{t}} \mathbf{x} \leq K, t=1, \ldots, M \\
& C_{6}: \mathbf{x}^{T} \mathbf{D}_{\mathbf{n}} \mathbf{x}=\Delta_{n}, n=1, \ldots, N \\
& C_{7}: \mathbf{x} \in\{0,1\}^{N M}
\end{aligned}
$$

where matrix $\mathbf{D}_{\mathbf{n}}$ and $\mathbf{E}_{\mathbf{t}}$ are diagonal matrix to select the $n$-th beam for every time slot and all the active beams at the $t$-th time slot, respectively. That is,

$$
\begin{aligned}
\mathbf{E}_{\mathbf{t}} & =\operatorname{diag}\left(\mathbf{b}_{t}\right) \otimes \mathbf{I}_{N} \\
\mathbf{D}_{\mathbf{n}} & =\mathbf{I}_{M} \otimes \operatorname{diag}\left(\mathbf{e}_{n}\right)
\end{aligned}
$$

where $\mathbf{b}_{t}$ is $\mathbf{a}(M \times 1)$ vector whose entries are 0 except for the $t$-th component which is 1 ; and $\mathbf{e}_{n}$ has been already defined in (8).

Problem $P_{2}$ corresponds to a Binary Quadratic Programming (BQP) [15], i.e. a problem involving a quadratic objective function with binary variables. What's more, it is easy to find out that $\mathbf{x}^{T} \mathbf{x}=\sum_{n=1}^{N} \Delta_{n}$ is a constant. So the objective function can always be modified to be positive semidefinite by adding constant. That is,

$$
\mathbf{x}^{T} \tilde{\mathbf{A}} \mathbf{x} \Leftarrow \mathbf{x}^{T}(\tilde{\mathbf{A}}+\lambda \mathbf{I}) \mathbf{x}
$$

where $\lambda$ is some value which is chosen to guarantee the convexity of the objective function. Typically, $\lambda$ is chosen to be the minus of the minimum eigenvalue of $\tilde{\mathbf{A}}$. 
The standard semidefinite relaxation of problem $P_{2}$ in (9) can be expressed as

$$
\begin{array}{rlr}
P_{3}: & \underset{\mathbf{x}}{\operatorname{minimize}} \operatorname{Tr}(\tilde{\mathbf{A}} \mathbf{X}) \\
\text { s.t. } & C_{8}: \operatorname{Tr}\left(\mathbf{E}_{\mathbf{t}} \mathbf{X}\right) \leq K & t=1, \ldots, M \\
& C_{9}: \operatorname{Tr}\left(\mathbf{D}_{\mathbf{n}} \mathbf{X}\right)=\Delta_{n} & n=1, \ldots, N \\
& C_{10}: \operatorname{diag}(\mathbf{X})=\mathbf{x} \\
& C_{11}:\left[\begin{array}{cc}
\mathbf{X} & \mathbf{x} \\
\mathbf{x}^{T} & 1
\end{array}\right] \succeq 0 \\
& C_{7}: \mathbf{x} \in\{0,1\}^{N M}
\end{array}
$$

which can be solved with advanced optimization toolboxes supporting mixed-integer models like CVX [16].

TABLE I: Simulation Parameters

\begin{tabular}{l|r}
\hline \hline Satellite Orbit & $13^{\circ} \mathrm{E}(\mathrm{GEO})$ \\
Satellite Total Transmit Power & $6000 \mathrm{~W}$ \\
OBO & $3 \mathrm{~dB}$ \\
Addition Payload Loss & $2 \mathrm{~dB}$ \\
Number of Virtual Beams, $N$ & 67 \\
Beam Radiation Pattern $\left(G_{k, l} e^{j \phi_{k, l}}\right)$ & Provided by ESA \\
Downlink Carrier Frequency & $19.5 \mathrm{GHz}$ \\
User Link Bandwidth, $B$ & $500 \mathrm{MHz}$ \\
Roll-off Factor & $20 \%$ \\
Number of time slots $(M)$ & 20 \\
\hline
\end{tabular}

\section{Simulation Results AND Discussion}

We consider a GEO satellite system with $N=67$ virtual beams. A summary of the simulation parameters is given in Table I. Unless mentioned otherwise, the beam demand $D_{n}$ is generated uniformly at random between 0 and $750 \mathrm{Mbps}$, according to the considered overall system capacity. The perbeam transmit power $P_{\text {beam }}$ is obtained by dividing the total radiated power between the total number of active beams per time-slot.

\section{A. Capacity-on-Demand Evaluation}

Fig. 2 compares the proposed technique to the benchmark schemes in terms of average per-beam supplied capacity matching the per-beam requested demand (in blue). In particular, we compare with the so-called Cluster Hopping $(\mathrm{CH})$ technique [12] where 2 independently precoded clusters of 6 beams each are activated at each time slot. In addition, we compare with a conventional $\mathrm{BH}$ technique which has been obtained based on the design in [12] but considering clusters of 1 beam each and without precoding. The average per-beam supplied capacity is calculated over 500 Monte Carlo simulations with different demand realizations. Fig. 2 demonstrates the superior performance of the proposed technique, which unlike the benchmarks, it is able to provide beam capacity following the requested demands. The $\mathrm{CH}$ technique suffers from the limitation of pre-defined clustering shapes, which unavoidable illuminate low-demand beams with high-demand beams. On the other hand, the conventional BH is shown to fall short in supplying enough capacity due to it is unable to illuminate high-demand areas at once.

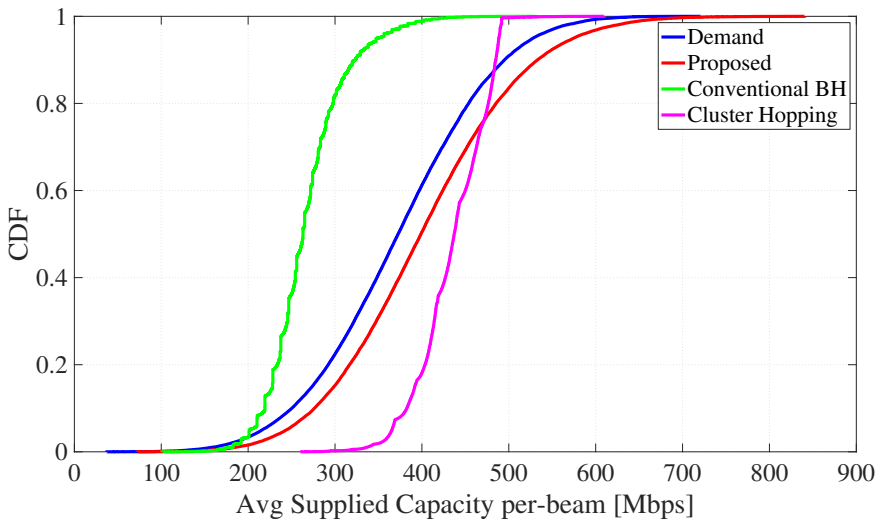

Fig. 2: Comparison of the proposed technique versus existing techniques in terms of per-beam demand matching.
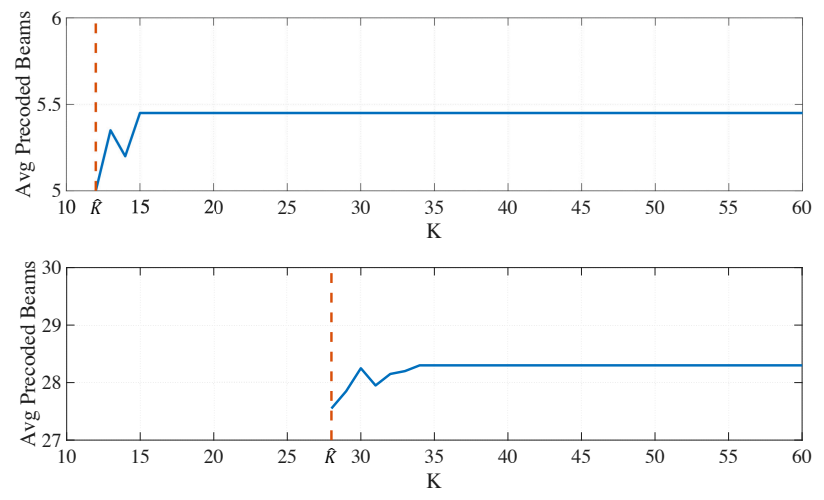

Fig. 3: Impact of maximum number of active beams $K$ : (a) Low-Demand Realization, (b) High-Demand Realization.

\section{B. Sensitivity Analysis: Max. Number of Active Beams K}

Fig. 3 illustrates the impact of variable $K$ in terms of the average number of beams that need to be precoded at each time instance. Fig. 3(a) corresponds to a low-demand scenario, where $D_{n}$ is generated uniformly at random between 0 and $240 \mathrm{Mbps}$, while Fig. 3(b) corresponds to a high-demand scenario, with $D_{n}$ between 0 and 640 Mbps.

Fig. 3 shows that there is a minimum value of $K$ to make $\left(P_{3}\right)$ feasible (indicated with a vertical red line), which we name $\hat{K}$. In fact, to satisfy the beam demands within $M$ time slots, the value of $\hat{K}$ can be obtained as $\hat{K}=\left\lceil\frac{\sum_{n} \Delta_{n}}{M}\right\rceil$. From Fig. 3, we can observe that the penalty remains constant independent of the value of $K \geq \hat{K}$. This is because the proposed algorithm minimizes the interference by equally distributing the number of active beams across the time window. As a consequence, for the remaining experiments, we fix $K=\hat{K}$.

\section{Sensitivity Analysis: System Demand}

Fig. 4 shows the evolution of the average number of beams that need to be precoded with the increasing overall system demand considering the proposed technique. As expected the number of precoded beams increases with the demand, 


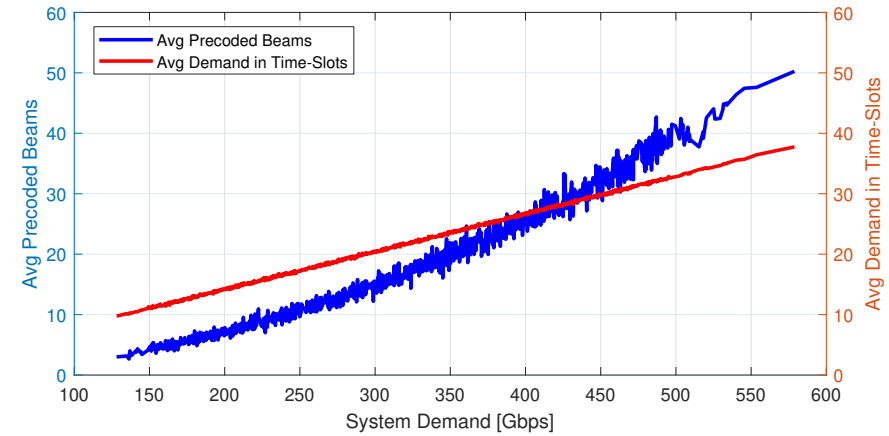

Fig. 4: Average number of precoded beams per time-slots and Average demand in number of time-slots versus overall system demand.

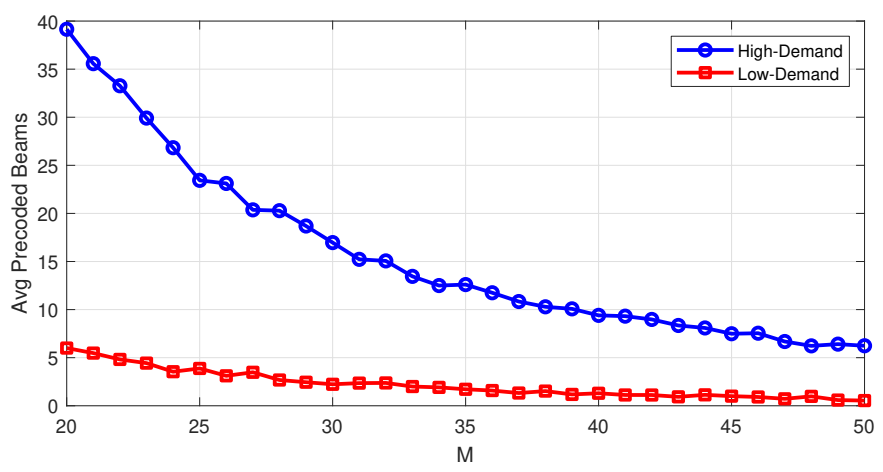

Fig. 5: Impact of time window length $M$ into the average number of precoded beams.

resulting in added system complexity. Also in Fig. 4, and for the sake of clarity, the red curve illustrates the average demand in number of time-slots (i.e. $\frac{1}{M} \sum_{n=1}^{N} \Delta_{n}$ ). As expected, this increases with the system demand.

\section{Sensitivity Analysis: Time Window}

Complementing the results presented in Fig. 4, Fig. 5 illustrates the average number of precoded beams corresponding to two scenarios considered in Fig. 3, i.e. "High-Demand" and "Low-Demand", versus the various values of window length $M$. As can be observed, the higher value of $M$ results in the lower average number of beams which are activated within each time slot in both scenarios.

\section{CONCLUSION}

In this paper, we proposed an algorithm to design the dynamic beam illumination pattern of a beam-hopping system focusing on the per-beam demand satisfaction. We have focused on the interference minimization such that the number of beams that need to be precoded are kept to minimal in an attempt to reduce system complexity. The preliminary evaluation has been promising, showing that the proposed algorithm outperforms the relevant benchmark schemes. Further research will be conducted by combining the proposed algorithm with current power control methods to improve the per-beam demand matching.

\section{ACKNOWLEDGMENT}

This work has been supported by the Luxembourg National Research Fund (FNR) under the project "FlexSAT: Resource Optimization for Next Generation of Flexible SATellite Payloads" (C19/IS/13696663).

\section{REFERENCES}

[1] NetWorld 2020, "White Paper: SatCom Resources for Smart andSustainable Networks and Services," https://www.networld2020.eu/satcom-wg/, 2019, [Online; accessed 07-Feb-2021].

[2] A. I. Perez-Neira, M. A. Vazquez, M. R. B. Shankar, S. Maleki, and S. Chatzinotas, "Signal processing for high-throughput satellites: Challenges in new interference-limited scenarios," IEEE Signal Processing Magazine, vol. 36, no. 4, pp. 112-131, 2019.

[3] H. Al-Hraishawi, E. Lagunas, and S. Chatzinotas, "Traffic simulator for multibeam satellite communication systems," in 2020 10th Advanced Satellite Multimedia Systems Conference and the 16th Signal Processing for Space Communications Workshop (ASMS/SPSC), 2020, pp. 1-8.

[4] O. Kodheli et al., "Satellite communications in the new space era: A survey and future challenges," IEEE Communications Surveys Tutorials, pp. 1-1, 2020.

[5] A. Freedman, D. Rainish, and Y. Gat, "Beam Hopping - How To Make it Possible," in Proc. of Ka and Broadband Communication Conference, Oct. 2015.

[6] R. Alegre-Godoy, N. Alagha, and M. A. Vázquez-Castro, "Offered capacity optimization mechanisms for multi-beam satellite systems," in 2012 IEEE International Conference on Communications (ICC), 2012, pp. 3180-3184.

[7] J. Anzalchi, A. Couchman, P. Gabellini, G. Gallinaro, L. D’Agristina, N. Alagha, and P. Angeletti, "Beam hopping in multi-beam broadband satellite systems: System simulation and performance comparison with non-hopped systems," in 2010 5th Advanced Satellite Multimedia Systems Conference and the 11th Signal Processing for Space Communications Workshop, 2010, pp. 248-255.

[8] Piero Angeletti, David Fernandez Prim, and Rita Rinaldo, "Beam hopping in multi-beam broadband satellite systems: System performance and payload architecture analysis," in 24th AIAA International Communications Satellite Systems Conference.

[9] G. Cocco, T. de Cola, M. Angelone, Z. Katona, and S. Erl, "Radio resource management optimization of flexible satellite payloads for $\mathrm{dvb}$ s2 systems," IEEE Transactions on Broadcasting, vol. 64, no. 2, pp. 266-280, 2018

[10] L. Lei, E. Lagunas, Y. Yuan, M. G. Kibria, S. Chatzinotas, and B. Ottersten, "Beam illumination pattern design in satellite networks: Learning and optimization for efficient beam hopping," IEEE Access, vol. 8, pp. 136655-136667, 2020.

[11] A. Ginesi, E. Re, and P.D. Arapoglou, "Joint beam hopping and precoding in hts systems," in 9th Int. Conf. on Wireless and Satellite Systems (WiSATS), 2017.

[12] M. G. Kibria, E. Lagunas, N. Maturo, D. Spano, and S. Chatzinotas, "Precoded cluster hopping in multi-beam high throughput satellite systems," in 2019 IEEE Global Communications Conference (GLOBECOM), 2019, pp. 1-6.

[13] C. B. Peel, B. M. Hochwald, and A. L. Swindlehurst, "A vector-perturbation technique for near-capacity multiantenna multiuser communication-part i: channel inversion and regularization," IEEE Transactions on Communications, vol. 53, no. 1, pp. 195-202, Jan 2005.

[14] M. A. Vazquez, A. Perez-Neira, D. Christopoulos, S. Chatzinotas, B. Ottersten, P. Arapoglou, A. Ginesi, and G. Tarocco, "Precoding in multibeam satellite communications: Present and future challenges," IEEE Wireless Communications, vol. 23, no. 6, pp. 88-95, December 2016.

[15] Otto Nissfolk, Binary Quadratic Optimization, Ph.D. thesis, Abo Akademi University, Finland, 2016.

[16] Michael Grant and Stephen Boyd, "CVX: Matlab software for disciplined convex programming, version 2.1," http://cvxr.com/cvx, Mar. 2014. 